\title{
Doege-Potter syndrome due to endothoracic solitary hypoglycemic fibrous tumor
}

Madaleine Lopez-Hinostroza ${ }^{1}$, Jeel Moya Salazar ${ }^{2}$, Juan Dávila ${ }^{1}$, Angélica Absencio ${ }^{1}$, and Hans Contreras-Pulache ${ }^{3}$

${ }^{1}$ Level IV Hospital Guillermo Almenara Irigoyen

${ }^{2}$ Hospital Nacional Docente Madre Nino San Bartolome

${ }^{3}$ Universidad Norbert Wiener

September 27, 2021

\begin{abstract}
Doege-Potter syndrome leads to severe and sustained symptomatic hypoglycemia and is associated with the solitary fibrous tumor. It is a rare cause and its diagnosis requires a clinical suspicion and other markers such as insulin-like growth factor-II. Here, we describe a case of a patient with intrathoracic tumor and hypoglycemia
\end{abstract}

Doege-Potter syndrome due to endothoracic solitary hypoglycemic fibrous tumor

Madaleine Lopez-Hinostroza ${ }^{1} *$, Jeel Moya-Salazar ${ }^{2,3}$ *, Juan Dávila ${ }^{1}$, Angélica Y. Absencio ${ }^{4}$, Hans Contreras-Pulache ${ }^{3}$

${ }^{1}$ Department of Respiratory Disease, Hospital Nacional Guillermo Almenara Irigoyen, Lima, Peru.

${ }^{2}$ Hospital Nacional Docente Madre Niño San Bartolomé, Lima, Peru

${ }^{3}$ South America Center for Education and Research in Public Health, Universidad Norbert Wiener, Lima, Peru

${ }^{4}$ Department of Pathology, Hospital Nacional Guillermo Almenara, Lima, Perú.

* These authors contributed equally

\section{Correspondence:}

Dr. Hans Contreras-Pulache, South America Center for Education and Research in Public Health, Universidad Norbert Wiener, Lima 51001, Peru. Email: hans.contreras@uwiener.edu.pe

Dr. Madaleine Lopez-Hinostroza, 1380 Costanera Avenue, 1502 Dept., San Miguel, Lima 15088, Peru. Email:

Running tittle: Solitary fibrous tumor causing Doege-Potter syndrome

\begin{abstract}
Doege-Potter syndrome leads to severe and sustained symptomatic hypoglycemia and is associated with the solitary fibrous tumor. It is a rare cause and its diagnosis requires a clinical suspicion and other markers such as insulin-like growth factor-II. Here, we describe a case of a patient with intrathoracic tumor and hypoglycemia.
\end{abstract}

Keywords: Doege-Potter syndrome, Solitary fibrous tumor, hypoglycemia, Non-islet cell tumor, Insulin-like growth factor II, Peru. 


\section{Key Clinical Message}

Doege-Potter syndrome is a rare cause of symptomatic, severe, and sustained hypoglycemia. This syndrome is associated with a solitary fibrous tumor and its diagnosis is based on an acute set of clinical characteristics and biological markers.

\section{Introduction}

Karl Doege and Roy Potter in 1930 described the presence of a non-beta-pancreatic cell tumor associated with hypoglycemia in a patient with a fibrous tumor in the mediastinum. Since then, the eponymous of these authors has been used to refer to the presence of an intrathoracic tumor associated with symptomatic, severe, and sustained hypoglycemia. The pathophysiological mechanisms that explain the hypoglycemic syndrome are glucose consumption by the tumor and excess secretion of insulin-like growth factor II (IGF-II), a protein that inhibits the release of glucose by the liver, and substances that inhibit its action or the secretion of counterinsular hormones, which leads to the failure of one of the mechanisms to prevent hypoglycemia. ${ }^{1}$

The solitary fibrous tumor (SFT) is a slow-growing, large tumor of mesenchymal origin that can cause Doege-Potter syndrome in about $2-4 \%$ of cases. ${ }^{2}$ The solitary fibrous tumor (SFT) is a slow-growing, large tumor of mesenchymal origin that can cause Doege-Potter syndrome in about 2-4\% of cases. SFT is difficult to distinguish from other tumors, but on chest tomography, it generally appears as a round and well-defined homogeneous mass, large tumors can reach more than $20 \mathrm{~cm}$ in diameter, and $90 \%$ of them have benign features. ${ }^{3}$

For its diagnosis, immunohistochemical markers such as CD34 + are used, which is strongly expressed by this tumor, to differentiate it from other cancerous tumors. Complete surgical resection to negative margins, even for tumors classified as high risk, given the low global metastatic potential and the lack of efficacious adjuvant therapy, is the mainstay of localized SFT therapy. ${ }^{3,4}$

Here, we describe for the first time an interesting case of a Peruvian patient who accidentally discovered an intrathoracic tumor of mesenchymal origin, accompanied by severe and persistent hypoglycemia.

\section{Case report}

A 74-year-old male patient with a history of hypertension 15 years ago, type 2 diabetes mellitus 20 years ago with metformin $850 \mathrm{mg}$ q.d treatment, presents with mMRC2 grade dyspnea, chest pain that has not radiated for two weeks before admission. Due to sudden loss of consciousness, he is taken by emergency, being immediately referred to the Shock Trauma Unit (Glasgow 10/15), to control vital functions: BP: 170/90 mmHg, HR: $90 \mathrm{bpm}$, FR: $26 \mathrm{rpm}$, SATO2: 95\%, FIO2: 21\%, and on preferential clinical examination: vesicular murmur is found abolished in the left hemithorax and preserved in the right hemithorax, no added sounds are heard.

We take capillary blood glucose $(38 \mathrm{mg} / \mathrm{dl})$, classifying it as acute encephalopathy without neurological targeting and symptomatic hypoglycemia. Glucose infusion serum treatment is administered, recovering consciousness without any neurological sequelae. A computerized axial tomography of the chest without contrast was performed, showing a mass and left pleural effusion (Fig. 1). The patient in his serial capillary glycemia controls presents episodes of symptomatic, sustained and persistent recurrent hypoglycemia, for which treatment with glucose serum is maintained. In hematic biometry controls we found a glycosylated hemoglobin (HbA1c): $5.7 \%$, basal cortisol $8 \mathrm{am}$ of $5.9 \mathrm{mg} / \mathrm{dl}$, insulin-like growth factor I (IGF I): $74 \mathrm{ng} / \mathrm{ml}$, peptide C: $0.01 \mathrm{ng} / \mathrm{dL}$, and basal insulin of $0.04 \mathrm{U} / \mathrm{I}$.

\section{Investigation}

A bronchofibroscopy was performed, finding direct signs of malignancy and stenosis of the left bronchial branch. For this reason, transbronchial biopsy samples of the left lung mass and bronchial lavage were taken, obtaining an SFT. The tumor immunohistochemistry results were BCL2 +, CD34 + focal, CD99 Focal cytoplasmic, Actin negative, S100 negative, EMA negative, Panker negative (Fig. 2), and Papanicolaou and 
Block cell with a positive result for malignant tumor cells. In addition, Bone scintigraphy was performed with a negative result for metastasis.

The patient continues to present symptomatic and recurrent hypoglycemia, considering endogenous hyperinsulinism in the differential diagnosis. Since the IGF II value was found to be 721 U/I, we considered non-island cell hypoglycemia and paraneoplastic syndromes of IGF-II-producing tumors as a definite diagnosis.

A pulmonary ventilation/perfusion scintigraphy was performed indicating absence of left lung perfusion and preserved in the right lung. A multidisciplinary medical meeting was carried out deciding to excise the lung tumor by the chest surgery team. Surgical intervention was performed 31 days after admission, and the operating time was 11 hours. Complete removal of the endothoracic tumor (weight: $3.1 \mathrm{~kg}$ ) was successfully achieved, which was sent for a pathological study and two left thoracic drainage tubes were placed: anterior and posterior (Fig. 3).

At $8.10 \mathrm{pm}$ on the same day, the patient presented cardiorespiratory arrest with asystole rhythm due to type III and IV acute respiratory failure, and haemorrhagic shock. Cardiopulmonary resuscitation was performed for 2 minutes with the administration of vasoconstrictor drugs and transfusion of 4 concentrated blood cells. The patient responded to extubation 2 days after the operation, and there were no sequelae of cardiorespiratory arrest. Five days later, both chest drainage tubes are removed. On the sixth day after surgery, the patient was discharged without re-presenting hypoglycemic episode after surgery. On evaluation by outpatient consultation 3 weeks after discharge, the chest radiograph shows pulmonary reexpansion in the left hemithorax (Figure 4).

\section{Discussion}

This report is one of the few in Peru ${ }^{7}$ and agrees with the clinical presentation reported by Han et al., ${ }^{4}$ who have reported that the most common cause of Doege-Potter syndrome is a benign solitary fibrous tumor involving the Right hemothorax associated with pleural damage. Han et al., reported 71 cases of Doege-Potter syndrome, 43 of which $(60.6 \%)$ were malignant, including SFT of the pelvis, bladder, and retroperitoneum associated with high-frequency hypoglycemia. ${ }^{4}$

The SFT associated with Doege-Potter syndrome reported in this 74-year-old patient has demonstrated positivity for BCL2 +, focal CD34 +, and Focal CD99 +. Mohammed et al., ${ }^{5}$ have reported a large number of lesions that are positive for CD34 + and CD99 +, as well as other case reports, have shown immunoreactivity of these cellular markers. ${ }^{6,7}$ However, the authors emphasize that these immunohistochemical markers do not have high specificity and may be present in many soft tissue neoplasms.

In this study, we reported negative for actin, S100 protein (S100), and epithelial membrane antigen (EMA), which is different from previous reports that showed positive for smooth muscle actin, EMA, S100, and keratin. Given this particular performance of these markers, STAT6 has turned out to be more specific, being positive in $>95 \%$ of the cases, recently reported as part of the pool of tests to address these cases. ${ }^{8}$

This clinical case has reported Doege-Potter syndrome secondary to the SFT tumor. Although the main paraneoplastic symptom of SFT is osteoarthropathy, hypoglycemia is less than $5 \%$, which is a rare complication. Hypoglycemia is usually secondary to the tumor overproducing IGF II precursor that leads to tumor growth, malignant transformation, and carcinogenesis. ${ }^{9}$ Our patient had an IGF II concentration of 721 U/I that exceeds the threshold of $10 \mathrm{U} / \mathrm{I}$, indicating an excess production of IGF II characteristic of non-islet cell tumors. ${ }^{4,9,10}$

Insulin is a 51-amino acid protein with hormone functions, and has mediators such as peptide $\mathrm{C}$ in its production process. This hormone is responsible for glycemic control, and in Doege-Potter syndrome it is common for insulin levels to be decreased. As in previous studies ${ }^{5,6}$ our patient had low levels of basal insulin (0.04 U / I) and C-peptide (0.01 ng / dL).

It has been described that the most frequent age of SFT is older adults. Several studies have reported SFT in patients aged 67 and 83 years, ${ }^{5,9,11}$ although there are case series in patients aged 48 years, ${ }^{12} 52$ years, ${ }^{8} 55$ 
years, ${ }^{6}$ and 58 years. ${ }^{3}$ In this study, an elderly patient (74 years old) with Doege-Potter syndrome secondary to benign SFT was reported for the first time in Peru, which is different from previous reports. ${ }^{6}$

The definitive underlying treatment for Doege-Potter syndrome secondary to benign SFT is palliative debulking, complete resection of the tumor mass, chemotherapy, cryoablation, radiofrequency ablation, or chemoembolization. Previous studies have described complete resection as an effective treatment for this disease, as seen in some cases, including the one we reported in this article. ${ }^{4,5,9}$ In addition, other surgical techniques with a favourable prognosis have been reported as successful. ${ }^{3,13}$

With the discovery of the NAB2-STAT6 gene fusion as the characteristic molecular driver of SFT, it is possible that embolization therapy and radiation will be effective. Based on this, individualized therapies aimed at inhibiting the descending pathways of NAB2-STAT6 could be considered for the treatment of this rare group of neoplasms. ${ }^{5}$

Finally, there is a growing interest in SFT and Doege-Potter syndrome. In Latin America, several cases have been reported in the Argentine, ${ }^{14,15}$ Peruvian, ${ }^{6}$ Chilean, ${ }^{16}$ Brazilian, ${ }^{8}$ Colombian,,${ }^{71,17,18}$ and Bolivian ${ }^{19}$ population, showing that even being a rare cause of the paraneoplastic syndrome is of great interest in clinical practice.

\section{Conclusion}

Doege-Potter syndrome associated with solitary fibrous tumor is a rare cause of the paraneoplastic complication. Key to its diagnosis is the quantification of IGF II, the glycaemic control profile, and immunohistochemical markers. The surgery has been effective and its follow-up is necessary, which generally presents a favourable prognosis.

\section{Authorship}

All authors contributed equally to this work.

\section{References}

1. van Houdt WJ, Westerveld CM, Vrijenhoek JE, et al. Prognosis of solitary fibrous tumors: a multicenter study. Ann Surg Oncol. 2013; 20(13):4090-5.

2. Klemperer P, Rabin CB. Primary neoplasms of the pleura: a report of five cases. Arch Pathol. 1931; 11:385.

3. Gold JS, Antonescu CR, Hajdu C, et al. Clinicopathologic correlates of solitary fibrous tumors. Cancer. 2002; 94(4):1057-68.

4. Han G, Zhang Z, Shen X, et al. Doege-Potter syndrome: A review of the literature including a new case report. Medicine (Baltimore). 2017; 96(27): e7417.

5. Mohammed T, Ozcan G, Siddique AS, Araneta III RN, Slater DE, Khan A. Doege-Potter Syndrome with a Benign Solitary Fibrous Tumor: A Case Report and Literature Review. Case Rep Oncol 2021; 14:470-476.

6. Pinto M, Morello E, Ramírez RM, Ramírez J, Cáceres J. Síndrome Doege-Potter. Reporte de caso. Rev Med Hered. 2013; 24:136-140.

7. Sandoval-Alzate HF, Parra-Gamboa JM, Angulo-Casalis A. Doege-Potter syndrome. Acta Med Colom. 2020; 45(3):1-4

8. Estradioto L, Araujo RB, Bergonse Neto N, Araújo VB, Coelho MS, Silva LLG. Doege-Potter syndrome: a rare presentation of a solitary fibrous tumor of the pleura. Braz J Oncol. 2021;17:

9. de Groot JW, Rikhof B, van Doorn J, et al. Non-islet cell tumour-induced hypoglycaemia: a review of the literature including two new cases. Endocr Relat Cancer. 2007;14(4):979-93.

10. Fukuda I, Hizuka N, Ishikawa Y, et al. Clinical features of insulin-like growth factor-II producing non-islet-cell tumor hypoglycemia. Growth Horm IGF Res. 2006; 16(4): 211-6.

11. Sandoval-Alzate HF, Parra-Gamboa JM, Angulo-Casalis A. Doege-Potter syndrome. Acta Med Colomb 2020; 45: 1-4. 
12. Chamberlain MH, Taggart DP. Solitary fibrous tumor associated with hypoglycemia: an example of the Doege-Potter syndrome. J Thorac Cardiovasc Surg. 2000; 119(1):185-7.

13. Solsi A, Pho K, Shojaie S, Findakly D, Noori T. Doege-Potter Syndrome and Pierre-Marie-Bamberger Syndrome in a Patient with Pleural Solitary Fibrous Tumor: A Rare Case with Literature Review. Cureus 12(5): e7919.

14. Soutelo J, Moldes S, Martin A, Lutfi R, Reyna ML. Hypoglycemia induced by a solitary fibrous tumor of the lung or Doege-Potter syndrome: Report of one case. Rev Med Chile. 2016; 144(1): 129-133.

15. Prado F, Dos Ramos JP, Larrañaga N, Espil G, Kozima S. Tumor Fibroso Solitario y síndrome DoegePotter. 2018; 78: 47-49.

16. Rodrigo CM, Roberto GL, René SF, Rodrigo KP, Emilio AC. Doege-Potter syndrome: hypoglycemia secondary to solitary fibrous tumor of the pleura. Report of one case. Rev Med Chile. 2012; 140: 353-357.

17. Fernández-Trujillo L, Bolaños JE, Álvarez C, et al. Doege-Potter Syndrome and Hypoglycemia associated with Solitary Fibrous Tumor of the Pleura: Two Case Reports. Clin Med Insights Circ Respir Pulm Med. 2020; 14: 1179548420964759.

18. Luján M, Mejía S, Rojas S, Mira S. Síndrome de DoegePotter. Tumor fibroso de pleura irresecable asociado a hipoglucemia. Acta Med Colomb 2009; 34: 185-188.

19. Flores CG, Aguila GMV, Lazo VA, Alarcón DM, Luna CM, Marconi PE. Síndrome de Doege-Potter", tumor fibroso solitario endotorácico hipoglicemiante, ¿cuándo y cómo lo diagnóstico? y ¿cuál es mi conducta final?: presentación de un caso y revisión de la literatura. Rev Med La Paz, 2019; 25(1); $48-57$.

Figures

Figure 1 Coronal section chest CT scan shows a mass with a neo-proliferative appearance of the left lung measuring $24.9 \mathrm{~cm} \times 15.8 \mathrm{~cm} \times 18.9 \mathrm{~cm}$, associated with ipsilateral laminar pleural effusion and mass effect on the contralateral mediastinal structures.

Figure 2 Solitary fibrous tumor, microscopy. Microscopy revealed a storiform pattern arranged in hypocellular and hypercellular areas, necrosis of $20 \%$ of the tumor, and 6 mitoses in 10 high-power fields. With the immunohistochemical markers it was possible to observe positivity for CD99, CD34, Bcl-2, with actin and S100 being negative. With these findings, the final diagnosis was a malignant solitary fibrous tumor. (a) Storiform spindle cell proliferation,(b) CD99 positive, (c) CD34 positive, (d)Bcl-2 positive (all in 100x).

Figure 3 Surgery processes. (a) complete removal of solitary fibrous tumor (b) placement of 02 anterior and posterior TDT in the left hemithorax. Solitary fibrous tumor, macroscopy. (c) Solid tumor $(25 \times 20 \times 15 \mathrm{~cm})$ lobulated weighing $3.1 \mathrm{~kg}$, areas of necrosis and haemorrhage were identified in the laminations. (d) Solid whitish-brown cut surface, with areas of necrosis and haemorrhage.

Figure 4 Control chest X-ray 3 weeks after discharge, showing lung expansion. 